\title{
DNA methylation analysis reveals distinct methylation signatures in pediatric germ cell tumors
}

\author{
James F Amatruda ${ }^{1,2,4^{*}}$, Julie A Ross ${ }^{5,6}$, Brock Christensen ${ }^{8}$, Nicholas J Fustino ${ }^{1,4}$, Kenneth S Chen ${ }^{1,4}$, \\ Anthony J Hooten ${ }^{6}$, Heather Nelson ${ }^{6,7}$, Jacquelyn K Kuriger ${ }^{6,7}$, Dinesh Rakheja ${ }^{3}$, A Lindsay Frazier ${ }^{9}$ \\ and Jenny N Poynter ${ }^{5,6}$
}

\begin{abstract}
Background: Aberrant DNA methylation is a prominent feature of many cancers, and may be especially relevant in germ cell tumors (GCTs) due to the extensive epigenetic reprogramming that occurs in the germ line during normal development.

Methods: We used the Illumina GoldenGate Cancer Methylation Panel to compare DNA methylation in the three main histologic subtypes of pediatric GCTs (germinoma, teratoma and yolk sac tumor (YST); $N=51$ ) and used recursively partitioned mixture models (RPMM) to test associations between methylation pattern and tumor and demographic characteristics. We identified genes and pathways that were differentially methylated using generalized linear models and Ingenuity Pathway Analysis. We also measured global DNA methylation at LINE1 elements and evaluated methylation at selected imprinted loci using pyrosequencing.

Results: Methylation patterns differed by tumor histology, with 18/19 YSTs forming a distinct methylation class. Four pathways showed significant enrichment for YSTs, including a human embryonic stem cell pluripotency pathway. We identified $190 \mathrm{CpG}$ loci with significant methylation differences in mature and immature teratomas $(q<0.05)$, including a number of CpGs in stem cell and pluripotency-related pathways. Both YST and germinoma showed significantly lower methylation at LINE1 elements compared with normal adjacent tissue while there was no difference between teratoma (mature and immature) and normal tissue. DNA methylation at imprinted loci differed significantly by tumor histology and location.

Conclusion: Understanding methylation patterns may identify the developmental stage at which the GCT arose and the at-risk period when environmental exposures could be most harmful. Further, identification of relevant genetic pathways could lead to the development of new targets for therapy.
\end{abstract}

Keywords: Germ Cell Tumor, Teratoma, DNA Methylation, Imprinting

\section{Background}

Aberrant DNA methylation has been implicated in the etiology of multiple types of cancer, and has the potential to be especially relevant in germ cell tumors (GCTs) due to extensive epigenetic reprogramming that occurs in the germ line and early embryo during normal

\footnotetext{
*Correspondence: james.amatruda@utsouthwestern.edu

'Department of Pediatrics, University of Texas Southwestern Medical Center, Dallas, TX 75390, USA

${ }^{2}$ Department of Molecular Biology, University of Texas Southwestern Medical Center, Dallas, TX 75390, USA

Full list of author information is available at the end of the article
}

development. Histologically, GCTs can be divided into germinomas and non-germinomas. Germinomas (GERs; also called seminomas in the testis and dysgerminomas in the ovary) are tumors of undifferentiated germ cells that retain markers of pluripotency. In contrast, non-germinomas undergo differentiation to resemble somatic-type tissues (teratomas) or extra-embryonic structures (yolk sac tumor (YST) and choriocarcinoma). Studies of testicular GCTs have suggested that global methylation patterns differentiate the main histologic subtypes, with seminomas exhibiting global DNA

\section{Biomed Central}


hypomethylation while nonseminomas exhibit higher levels of methylation [1-3]. Initially, these data supported a theory that the methylation status indicated the embryonic stage of development of the primordial germ cell (PGC) when the tumor arose, with seminomas arising from a hypomethylated PGC and nonseminomas originating following de novo methylation of PGCs [1]. However, the hypomethylation observed in IGCNU (Intratubular Germ Cell Neoplasia, Unspecified), which is believed to be the precursor of both seminomas and non-seminomas, would suggest that both seminomas and nonseminomas are derived from a hypomethylated PGC [2]. Importantly, these alterations in methylation may be clinically relevant as DNA methylation has been shown to predict response to cisplatin treatment in an adult testicular cancer cell line [4].

Few studies have evaluated DNA methylation in pediatric GCTs [5-9]. Of these, three have identified hypermethylation in the promoter of tumor suppressor genes [6-8] while two others have identified unique methylation patterns that can help distinguish between tumors of different histologic subtypes [5,9]. In addition, alterations in genomic imprinting, which is controlled by DNA methylation, have been identified in GCTs [10-12].

In adolescents, as in adults, GCTs can present as germinomas, non-germinomas or a mixture of the two types. Young children less than 5 years of age, in contrast, develop primarily yolk sac tumors and teratomas. While yolk sac tumors are malignant at any age, the significance and clinical management of teratomas remain controversial. Mature teratomas contain fully differentiated tissues, and when occurring in the testis of prepubertal males or in the ovary are benign tumors [13]. In contrast, immature teratomas are characterized histologically by the presence of immature tissues, especially neural tissue. Higher-grade immature teratomas (those containing a higher percentage of immature elements) are often considered malignant and treated with cytotoxic chemotherapy [14]. While studies have identified clinical [15] and radiographic [16,17] features that separate mature from immature teratomas, the molecular signature of immature teratomas is not well understood. To date, methylation patterns have not been compared in mature and immature teratomas in the pediatric age group.

Given the important role of epigenetic reprogramming in normal germ cell development, additional studies of DNA methylation are likely to increase our understanding of the etiology of pediatric GCTs. In this analysis, we evaluated differences in DNA methylation in cancerrelated and imprinted genes by tumor and patient characteristics in a series of 51 pediatric GCTs, including YSTs, germinomas and teratomas (mature and immature). In addition, we evaluated global hypomethylation at LINE1 elements in a subset of the samples.

\section{Methods}

\section{Study samples}

GCTs from pediatric and adolescent patients (ages 0-21 years) were obtained from the Cooperative Human Tissue Network (Columbus, $\mathrm{OH}$ ) and from Children's Medical Center Dallas (CMC). Tumors were resected at initial diagnosis and snap frozen at $-70^{\circ} \mathrm{C}$. Pathology reports were also provided. Data were available for tumor histology, tumor location (gonadal or extragonadal), sex, and age at diagnosis. Normal adjacent tissue was also available for five of the tumors (four ovarian and one testicular) in our case series. Diagnosis was verified by a pediatric pathologist prior to molecular analysis and only samples with $>70 \%$ tumor cellularity of pure histological subtypes were included.

This analysis used existing data with no personal identifiers; therefore, the study was deemed exempt from review by the Institutional Review Boards of the University of Minnesota and the University of Texas Southwestern Medical Center and CMC.

\section{DNA extraction and bisulfite conversion}

Genomic DNA was isolated from GCT tissue and paired normal adjacent tissue (when available) using either the TRIzol $^{\circledR}$ extraction method (Invitrogen Life Technologies, California) or a QIAamp DNA Mini Kit (Qiagen Sciences, Maryland) according to the manufacturer's recommended protocol. DNA yield was quantified using $1 \mu \mathrm{l}$ DNA on a NanoDrop ${ }^{\mathrm{Ts}}$ spectrophotometer (Thermo Scientific, Maryland). Extracted DNA was stored at $-80^{\circ} \mathrm{C}$ until further analysis.

Prior to methylation analysis, $1 \mu \mathrm{g}$ genomic DNA was treated with sodium bisulfite to convert unmethylated cytosines to uracil using the EZ DNA Methylation Kit (Zymo Research, Orange, CA) according to manufacturer's protocol.

\section{GoldenGate cancer methylation panel}

DNA methylation at 1505 CpG loci in 807 cancerrelated genes was evaluated using the GoldenGate Cancer Methylation Panel I (Illumina, Inc.) in the Biomedical Genomics Center at the University of Minnesota following the manufacturer's protocol as described [18]. Replicates were included, including four duplicates that were included on both arrays and five duplicates that were included within one array.

\section{Pyrosequencing}

Array methylation results were validated by Pyrosequencing using a PyroMark MD80 Pyrosequencer (Qiagen) in a subset of the samples $(\mathrm{N}=41$ samples from $\mathrm{CHTN})$. Five pyrosequencing assays were designed for regions targeting the $\mathrm{CpG}$ loci on the array that had significant methylation differences between yolk sac tumor and 
other histologic subtypes. Briefly, PCR primers and sequencing primers were designed using PSQ Assay Design software (Qiagen, Inc) to capture the array CpG and as many neighboring CpGs as possible. Methylation at imprinted loci was evaluated using assays described in Woodfine et al. [19]. Primers and conditions are available upon request. Global LINE1 methylation was measured by pyrosequencing $4 \mathrm{CpG}$ loci in the LINE1 region as previously described [20]. LINE1 was measured in triplicate for each sample.

Commercially available Epitect methylated and unmethylated DNA standards were used as controls (Qiagen). In addition, a sequencing primer control and a no template control were included for each assay. The level of methylation for each $\mathrm{CpG}$ within the target region of analysis was quantified using the Pyro Q-CpG Software.

\section{Preparation of total RNA}

Total RNA was prepared from fresh frozen tumor tissue. 30-50 mg of tissue was homogenized using Tissue Miser (Fisher Scientific, Pittsburgh, PA) in TRIzol $^{\odot}$ Reagent (Invitrogen, Carlsbad, CA); approximately $1 \mathrm{~mL} \mathrm{TRIzol}^{\odot}$ per $50 \mathrm{mg}$ of tissue was used. After incubation for 30 minutes at room temperature, phase separation was done using chloroform $\left(200 \mu \mathrm{L} / 1 \mathrm{~mL}\right.$ Trizol $\left.{ }^{\circledR}\right)$. Sample was shaken vigorously, centrifuged at $13000 \mathrm{rpm}$ at $4^{\circ} \mathrm{C}$, and aqueous phase removed. RNA precipitation was done using $70 \%$ ethanol. To remove contaminant genomic DNA, on-column DNase digestion was done using RNase-Free DNase Digestion Kit (Qiagen, Valencia, CA). RNA isolation was done per manufacturer's instructions using RNeasy ${ }^{\odot}$ Mini Kit (Qiagen, Valencia, CA) and final elution performed in $20 \mu \mathrm{L} \mathrm{H}_{2} \mathrm{O}$. Quantity and purity

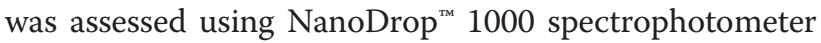
(Thermo Fisher Scientific, Wilmington, DE). Absorbance ratios at 260/280 $\mathrm{nm}$ and 260/230 $\mathrm{nm}$ were used to verify purity. Quality was further assessed by visualization of $28 \mathrm{~S}$ and $18 \mathrm{~S}$ bands after performing gel electrophoresis (1\% agarose in 1X Tris-EDTA-Acetate Buffer).

\section{Quantitative RT-PCR}

cDNAs were synthesized from $1 \mu \mathrm{g}$ of purified RNA using $\mathrm{RT}^{2}$ First Strand Kit (SABiosciences, Frederick, MD). Real-time quantitative PCR gene expression profiling was performed using a Wnt pathway-specific array (SABiosciences, Frederick, MD). Arrays profiled 84 pathway-specific genes with validated primers and contained internal control primers to assess genomic DNA contamination, RNA quality, and PCR amplification efficacy. RT-qPCR was performed on Applied Biosystems 7500 Real-Time PCR System (Carlsbad, CA) using RT $^{2}$ $\mathrm{SYBR}^{\odot}$ Green qPCR Master Mix (SABiosciences, Frederick, MD) as a fluorophore for amplicon detection. PCR conditions were as follows: $95^{\circ} \mathrm{C} \times 10$ minutes, $95^{\circ} \mathrm{C}$ for
15 seconds then $60^{\circ} \mathrm{C}$ for 1 minute $\times 40$ cycles, followed by a dissociation stage per manufacturer's protocol. Gene expression was normalized to endogenous HPRT, $\beta$-actin (ACTB) and glyceraldehyde-3-phosphate dehydrogenase (GAPDH), as these internal reference genes exhibited the least variation among the five internal reference genes evaluated. Fold change of gene expression was determined using the $2^{(-\Delta \Delta \mathrm{Ct})}$ method, and compared yolk sac tumors $(\mathrm{n}=4)$ to germinomas $(\mathrm{n}=3)$. We performed unsupervised hierarchical cluster analysis using web-based PCR data analysis software (www.sabiosciences.com/pcrarray dataanalysis.php). Raw gene expression data and calculations are shown in Additional file 1: Tables S2-S8, . Gene expression among histologic subtypes was compared using a type $3 t$-test (Additional file 1: Table S7).

Real time quantitative RT-PCR for SOX2 and DNMT3B $(\mathrm{N}=34$ samples $)$ was measured using a human embryonic stem cell PCR array (SA Biosciences). Fold change of gene expression was determined using the $2^{(-\Delta \Delta \mathrm{Ct})}$ method, and differences by tumor histology were measured using generalized linear models.

\section{Statistical analysis}

To understand differences in methylation patterns by tumor histology, we evaluated the three main histologic subtypes as determined by pathology review (YSTs, dysgerminomas, and teratomas) using the analytic techniques described below.

\section{GoldenGate methylation data}

Using the GoldenGate array, the methylation status of a CpG site is calculated as the variable $\beta$, which is the ratio of the fluorescent signal from the methylated allele to the sum of the fluorescent signals of both methylated and unmethylated alleles [18]. These values range from 0 (unmethylated) to 1 (fully methylated). GenomeStudio software (Illumina, Inc) was used to calculate the average methylation values $(\beta)$ from the $\sim 30$ replicate methylation measurements for each $\mathrm{CpG}$ locus. We used raw average $\beta$ values without normalization. GenomeStudio software was also used to assess data quality for each CpG loci. We omitted all CpG loci where $\geq 25 \%$ of the samples had a detection p-value $>0.05(\mathrm{~N}=16,1 \%)$. $\mathrm{X}$-linked CpG loci $(\mathrm{N}=84)$ were also removed, resulting in 1,405 loci for analysis.

The remaining analyses for the array data were conducted in R [21]. Methylation differences were evaluated using unsupervised hierarchical clustering with the Manhattan metric and average linkage as previously described [22]. We used recursively partitioned mixture modeling (RPMM) to test associations between methylation status and tumor (histology and location) and demographic (age at diagnosis and sex) characteristics as described [23] and implemented [22,24]. Briefly, samples 
are assigned to a methylation class using a model-based form of unsupervised clustering. Permutation-based tests (with 10,000 permutations) were used to test for associations between methylation class and covariates: we used a chi-squared test for categorical covariates (tumor histology, tumor location, and sex), and a Kruskal-Wallis test statistic to test associations between methylation class and age.

We then used a series of generalized linear models (GLM) to identify genes that were differentially methylated in YSTs and teratomas as previously described [22]. We accounted for multiple testing by controlling the false-discovery rate (FDR) [25]. Q-values were computed using the q-value package in $\mathrm{R}$.

Ingenuity Pathway Analysis (IPA; Ingenuity Systems) was used to identify pathways that were enriched in the list of CpG loci with significantly different methylation in YSTs compared with other histologic subtypes of tumors and in immature teratomas compared with mature teratomas. We implemented an IPA Core analysis with HUGO gene symbol as the identifier. For the analysis of YSTs, we restricted the analysis to CpG loci with up-regulated methylation (effect size > 1.0). For the comparison of mature and immature teratomas, we restricted the analysis to CpG loci with down-regulated methylation in immature teratomas. Both analyses included only CpG loci that were significant after controlling for multiple comparisons (q-value $<0.05$ )

\section{Pyrosequencing data}

Analysis of pyrosequencing data was conducted using SAS v. 9.2 (SAS Institute, Cary, NC). For the array validation assays, Pearson correlation coefficients and p-values are reported for correlation between Pyrosequencing and GoldenGate data.

For the imprinted loci, we would expect methylation to be $\sim 50 \%$. We categorized samples into three groups: 1 ) $<33 \%$ methylation (hypomethylated), 2) 33-66\% methylation (median methylation), and 3) $>66 \%$ methylation (hypermethylation) as previously described [11,26]. A Fisher's exact test was used to evaluate statistical significance of any differences in methylation by tumor histology and location.

Global LINE1 measure was evaluated by calculating the mean methylation level across the 4 LINE1 CpG loci. The mean was then averaged across the three replicates for each sample. Differences in LINE1 methylation across tumor histology (YST, germinoma, mature teratoma, immature teratoma, normal adjacent), tumor location, sex and age group were evaluated using a GLM with LINE1 methylation as the outcome variable.

\section{Results}

\section{Characteristics of the study samples}

Tumor specimens from 51 cases of pediatric GCT ranging in age from $0-21$ years were included in this analysis, including 19 yolk sac tumors (YSTs), 22 teratomas (8 immature and 14 mature), and 10 germinomas (Table 1 ). The YSTs were evenly distributed among boys and girls while the majority of cases with a germinoma or teratoma were female. Information on race/ethnicity was not available for the cases. Normal adjacent DNA was available for five cases (four ovary and 1 testis). Correlation coefficients for replicates were $\geq 0.95$ for all samples. There were no significant differences in methylation values when we compared samples extracted by the Trizol method with samples extracted by QIAamp after adjustment for tumor histology $(p>0.05)$.

\section{Methylation differences by tumor histology}

Unsupervised clustering of methylation data revealed differences by tumor histology (Figure 1). Modeling the methylation data with RPMM resulted in 8 methylation classes (Figure 2). Methylation classes were significantly associated with tumor histology $(\mathrm{p}<0.0001)$ : class 8 included 18/19 YSTs and classes 4-6 included all germinomas (Figure 1). Eight of the mature teratomas comprised their own methylation class (Class 3) while the remaining six were classified with either immature teratomas or dysgerminomas. Methylation class was also significantly associated with tumor location $(\mathrm{p}=0.005)$, sex $(p=0.008)$ and age at diagnosis $(p<0.001)$.

In comparisons of YSTs with the other histologic types, we identified $703 \mathrm{CpG}$ sites with statistically significant differences in methylation (q-value $<0.05$ ). Of the 233 CpGs most significantly associated with YST histology (q-value $<2.2 \mathrm{E}-16$ ), the majority (96\%) had increased methylation. Twenty-three CpG loci with the most significant $\mathrm{q}$ values also had an adjusted fold change in $\beta \geq 2.75$, indicating that YSTs had methylation levels $\geq 2.75$ times higher than tumors of other histologic types at these loci (Table 2).

We selected 5 CpG loci with significant methylation differences by tumor histology (q-value $<2.2 \mathrm{E}-16$ and foldchange $>2.50)$ for validation by Pyrosequencing (HOXA 9_E252_R, SOX1_P294_F, WT1_E32_F, WNT2_P217_F, MDR1_seq_42_S300_R). Array methylation was significantly correlated with Pyrosequencing methylation for all CpG loci (HOXA9: $r=0.92, p<0.0001$; SOX1: $r=0.92$, $\mathrm{p}<0.0001 ;$ WT1: $\mathrm{r}=0.93, \mathrm{p}<0.0001 ;$ WNT2: $\mathrm{r}=0.97$, $\mathrm{p}<0.0001 ;$ MDR1: $\mathrm{r}=0.97, \mathrm{p}<0.0001)$.

Using an Ingenuity Core Pathway Analysis, the human embryonic stem cell pluripotency $(p=0.02)$, embryonic stem cell differentiation into cardiac lineages $(\mathrm{p}=0.04)$, serotonin receptor signaling $(\mathrm{p}=0.04)$, and role of Wnt/GSK-3 $\beta$ signaling in the pathogenesis of influenza 
Table 1 Selected characteristics of the study samples

\begin{tabular}{|c|c|c|c|c|}
\hline & $\begin{array}{c}\text { Yolk Sac Tumor } \\
\text { N (\%) }\end{array}$ & $\begin{array}{l}\text { Immature Teratoma } \\
\text { N (\%) }\end{array}$ & $\begin{array}{c}\text { Mature Teratoma } \\
\text { N (\%) }\end{array}$ & $\begin{array}{c}\text { Germinoma } \\
\mathrm{N}(\%)\end{array}$ \\
\hline Total & 19 & 8 & 14 & 10 \\
\hline \multicolumn{5}{|l|}{ Age } \\
\hline Median (range) & $1(0-19)$ & $5(0-21)$ & $4.5(0-15)$ & $12(7-17)$ \\
\hline \multicolumn{5}{|l|}{ Sex } \\
\hline Male & $10(53)$ & $1(87)$ & $4(29)$ & 0 \\
\hline Female & $9(47)$ & $7(12)$ & $10(71)$ & $10(100)$ \\
\hline \multicolumn{5}{|l|}{ Tumor location } \\
\hline Ovary & $4(21)$ & $4(50)$ & $6(43)$ & $10(100)$ \\
\hline Testis & $6(32)$ & $1(12)$ & 0 & 0 \\
\hline Extragonadal & $9(47)$ & $3(37)$ & $8(57)$ & 0 \\
\hline
\end{tabular}

$(\mathrm{p}=0.05)$ pathways were enriched in CpG loci that had significantly higher methylation in YSTs compared with the other histologic types (q-value $<0.05$, fold change $>1.0$ ). Of these, the human embryonic stem cell pathway contains a number of genes that are highly relevant in germ cell biology (TCF4, WNT10B, BDNF, FGF2, BMP3, FZD9, WNT2, APC, SOX2, NTRK2, NTRK3, TGFB3, TGFB2, WNT1, PDGFRB). All of these genes had increased methylation in YST compared to other histologic subtypes, with $9 / 15$ genes showing a greater than 2 -fold increase (data not shown).

To determine if differential methylation of Wnt pathway genes affected the expression of the Wnt pathway in pediatric GCTs, we prepared RNA from fresh-frozen specimens of 7 of the tumors and performed quantitative RT-PCR of selected Wnt pathway genes (15 genes representing 25 methylated loci). Despite the fact that YSTs in general showed higher levels of methylation, of

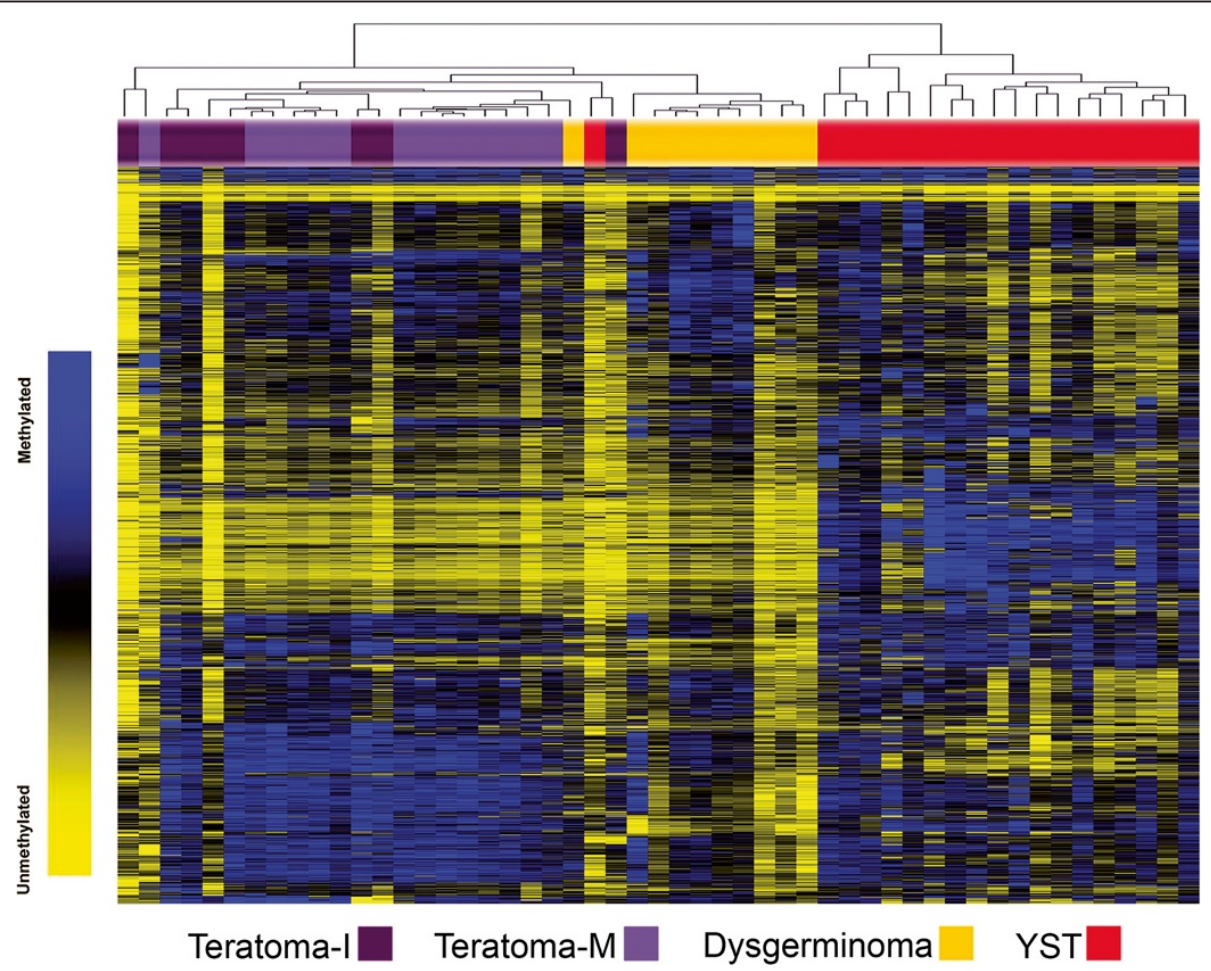

Figure 1 Unsupervised hierarchical clustering of CpG methylation in GCTs by tumor histology. Heat map from unsupervised hierarchical clustering based on Manhattan distance and average linkage of the 1404 autosomal CpG loci that passed initial quality control checks. Colored bars represent histologic subtype of the tumor. Light purple represents mature teratoma, dark purple represents immature teratoma, orange represents germinoma and red represents yolk sac tumor. Samples are in columns $(N=51)$ and $\mathrm{CpG}$ loci are in rows. Blue indicates high level of methylation (51-100\%), black equals 50\% methylation, and yellow indicates low level of methylation (0-49\%). 


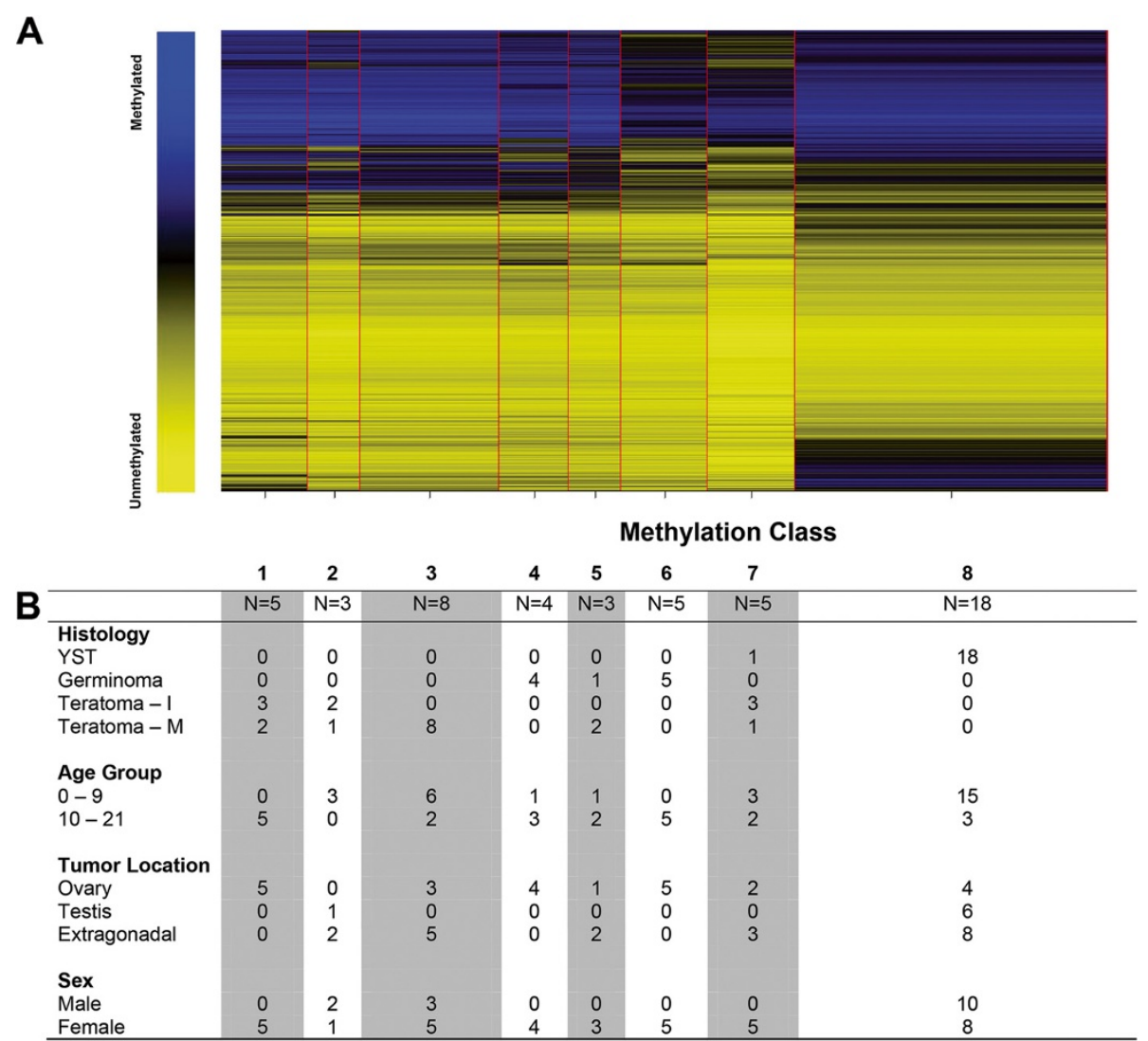

Figure 2 Recursively partitioned mixture model (RPMM) of CpG methylation in GCTs. A. Columns represent methylation class generated by RPMM and rows represent the average methylation within the class at each CpG site. Blue represents methylated and yellow represents unmethylated. The width of the row is proportional to the number of samples included in the methylation class. B. Characteristics of the tumors in each methylation class.

the 15 genes assessed 8 showed both lower levels of methylation and higher expression in YSTs compared to GER (Figure 3A; Additional file 1: Table S1). To further understand the transcriptional landscape of Wnt pathway activation in GCTs, we profiled a total of 84 genes comprising ligands, receptors, effectors and transcriptional targets in the Wnt pathway. Unsupervised clustering based on differential gene expression segregated YSTs and GERs and indicated higher levels of Wnt pathway gene expression in YSTs (Figure 3B; Additonal file 1: Tables S2-S8, Thus the Wnt pathway is active in YSTs and this activity may be explained at least in part by differential methylation.

Comparison of methylation in normal and tumor samples Paired normal adjacent tissue was also available for five tumors (2 dysgerminomas, 2 YSTs, and 1 teratoma). While the small sample size limits our ability to perform robust statistical analyses, the correlation coefficient for methylation $\beta$ values was higher for paired normal/ germinoma samples (0.87 and 0.92) and normal/teratoma (0.98) than for paired normal/YST (0.57 and 0.62).
Using a change in $\beta(\Delta \beta)>0.20$ to designate a significant difference in methylation between normal and tumor, we found that 425 and $428 \mathrm{CpG}$ loci were differentially methylated in the paired YST samples while 239 and 160 were differentially methylated in the paired dysgerminoma samples and only 15 were differentially methylated in the paired teratoma sample. The $\Delta \beta$ for the paired YST samples was large for the 23 genes that had the largest fold change in the comparison by tumor histology ( $\Delta \beta$ for paired samples shown in Table 2), suggesting that methylation at these $\mathrm{CpG}$ loci also distinguishes YST from normal testis or ovarian tissue.

\section{Comparison of mature and immature teratomas}

The molecular differences between mature and immature pediatric teratomas have not been explored. When we used RPMM to evaluate methylation differences only among the teratomas, tumor histology was not significantly associated with methylation class $(p=0.11)$. We also did not see significant differences by sex $(\mathrm{p}=0.10)$, tumor location $(\mathrm{p}=0.13)$ or age $(\mathrm{p}=0.28)$. When we evaluated the individual CpG loci, we identified 190 
Table 2 Top 23 genes with differential methylation in YST

\begin{tabular}{|c|c|c|c|c|}
\hline CpG Locus & Effect size $^{a}$ & q-value & $\Delta$ beta YST1 ${ }^{\mathrm{b}}$ & $\Delta$ beta YST2 ${ }^{\mathrm{b}}$ \\
\hline HLA.F_E402_F & 3.69 & $<2.2 \mathrm{E}-16$ & 0.86 & 0.81 \\
\hline WT1_E32_F & 3.41 & $<2.2 \mathrm{E}-16$ & 0.86 & 0.83 \\
\hline RASSF1_E116_F & 3.16 & $<2.2 \mathrm{E}-16$ & 0.87 & 0.79 \\
\hline CYP1B1_E83_R & 3.13 & $<2.2 \mathrm{E}-16$ & 0.74 & 0.54 \\
\hline CCNA1_E7_F & 3.13 & $<2.2 \mathrm{E}-16$ & 0.67 & 0.78 \\
\hline SLC22A3_E122_R & 3.12 & $<2.2 \mathrm{E}-16$ & 0.82 & 0.74 \\
\hline SCGB3A1_E55_R & 3.05 & $<2.2 \mathrm{E}-16$ & 0.80 & 0.77 \\
\hline HOXA9_E252_R & 2.99 & $<2.2 \mathrm{E}-16$ & 0.78 & 0.76 \\
\hline TFAP2C_E260_F & 2.98 & $<2.2 \mathrm{E}-16$ & 0.81 & 0.71 \\
\hline FGF3_P171_R & 2.96 & $<2.2 \mathrm{E}-16$ & 0.78 & 0.75 \\
\hline PDGFRB_P343_F & 2.95 & $<2.2 \mathrm{E}-16$ & 0.67 & 0.55 \\
\hline NPY_P295_F & 2.92 & $<2.2 \mathrm{E}-16$ & 0.81 & 0.75 \\
\hline ASCL2_P360_F & 2.90 & $<2.2 \mathrm{E}-16$ & 0.82 & 0.70 \\
\hline LRRC32_P865_R & 2.90 & $<2.2 \mathrm{E}-16$ & 0.81 & 0.44 \\
\hline CDK10_E74_F & 2.88 & $<2.2 \mathrm{E}-16$ & 0.83 & 0.65 \\
\hline HFE_E273_R & 2.87 & $<2.2 \mathrm{E}-16$ & 0.80 & 0.75 \\
\hline SOX1_P294_F & 2.86 & $<2.2 \mathrm{E}-16$ & 0.79 & 0.70 \\
\hline TAL1_P594_F & 2.83 & $<2.2 \mathrm{E}-16$ & 0.78 & 0.73 \\
\hline RASGRF1_E16_F & 2.80 & $<2.2 \mathrm{E}-16$ & 0.64 & 0.73 \\
\hline WT1_P853_F & 2.79 & $<2.2 \mathrm{E}-16$ & 0.78 & 0.73 \\
\hline$H L F \_E 192 \_F$ & 2.77 & $<2.2 \mathrm{E}-16$ & 0.80 & 0.75 \\
\hline GUCY2D_E419_R & 2.75 & $<2.2 \mathrm{E}-16$ & 0.83 & 0.06 \\
\hline HS3ST2_E145_R & 2.75 & $<2.2 \mathrm{E}-16$ & 0.84 & 0.79 \\
\hline
\end{tabular}

${ }^{a}$ Indicates the adjusted fold change in the $\beta$ value in the YST compared with the other histologic subtypes of GCT.

${ }^{b}$ Indicates the change in the $\beta$ value in the tumor sample compared to the paired normal adjacent in the two YST with available normal tissue.
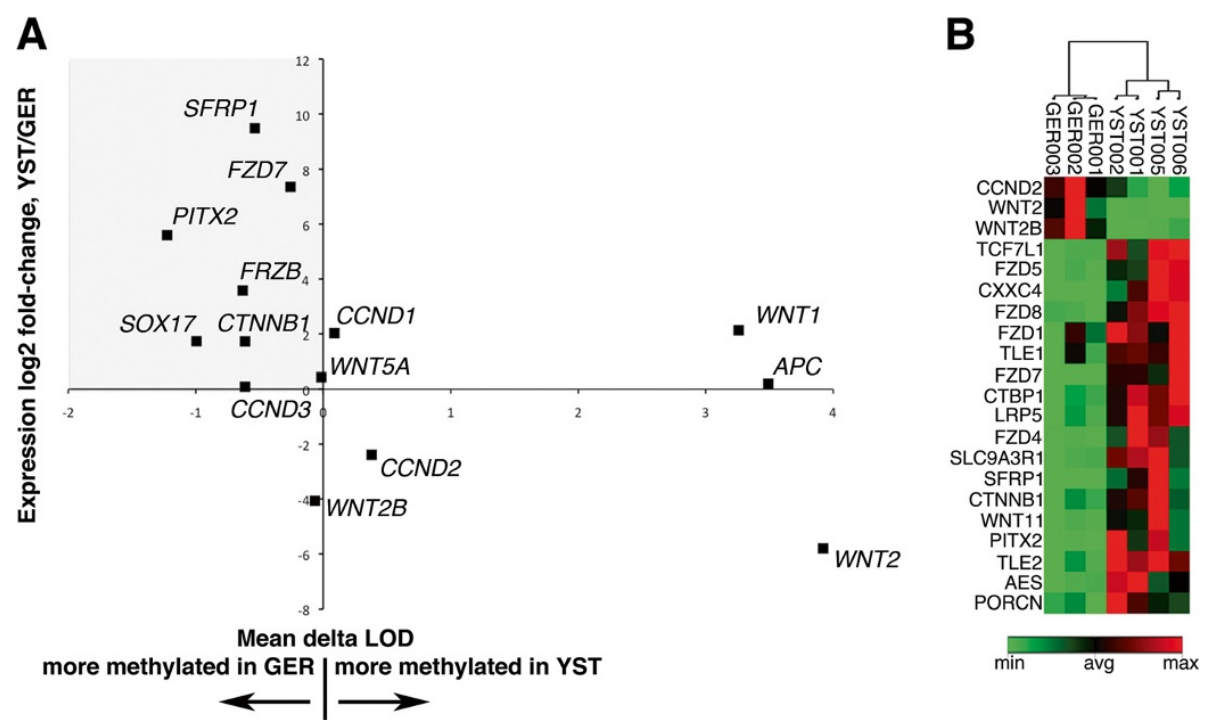

Figure 3 Correlation of methylation status and expression level for selected Wnt pathway genes. A. Log $_{2}$ fold-change in expression of selected Wht pathway genes in GER compared to YST plotted as a function of methylation level (expressed as the mean delta LOD). Of the 24 genes profiles, 12 exhibit higher expression and less methylation in YSTs (gray rectangle). B. Unsupervised clustering of Wnt pathway gene expression in pediatric gem cell tumors. The genes shown are differentially expressed in germinomas compared to yolk sac tumors ( $p \leq 0.05$ by two-tailed t-test). Red indicates high expression and green low expression. 
CpG loci with significant methylation differences after correction for multiple testing. Of these, the majority (96\%) had lower methylation in immature teratomas compared with mature teratomas. Using an Ingenuity Core Pathway Analysis, we identified 13 overlapping pathways enriched in CpG loci that had significantly reduced methylation in immature teratomas compared with mature teratomas (Table 3), including a number of pathways related to stem cell biology.

Notably, SOX2 was included in four of the pathways that differed between mature and immature teratomas. We were able to evaluate SOX2 by quantitative RT-PCR in 34 of the samples included in our analysis $(\mathrm{N}=17$ teratomas). Overall, we found that methylation at SOX2 was negatively correlated with expression $(r=-0.40$, $\mathrm{p}=0.06$ ). We also found that SOX2 expression varied by histologic subtype, with YST and germinoma having lower levels of expression than either group of teratomas, although this difference did not reach statistical significance ( $p=0.18$, Additonal file 1: Table S9). We also evaluated expression of DNMT3B, a known regulator of de novo methylation. We observed significantly higher levels of DNMT3B expression in YST compared with all other histologic subtypes $(\mathrm{p}<0.0001)$.

\section{Global LINE1 Methylation}

Global methylation at CpG loci in LINE1 elements was measured in a subset of the samples from the CHTN $(\mathrm{N}=41)$. We observed significant differences by tumor histology, with both YST (average methylation $=66 \%$, standard deviation (SD) 10\%) and dysgerminomas (average methylation $=42 \%$, SD $14 \%$ ) exhibiting significantly lower methylation levels than normal adjacent (average methylation $=82 \%, \mathrm{SD} 5 \%$ ), mature teratomas (average methylation $=78 \%$, SD 5\%), and immature teratomas (average methylation $=76 \%$, SD 11\%) $(\mathrm{p}<0.0001)$. No significant differences in average LINE1 methylation were observed by tumor location $(\mathrm{p}=0.39)$, sex $(\mathrm{p}=0.82)$ or age group $(\mathrm{p}=0.36)$.

\section{Methylation in imprinted genes}

Lastly, methylation in the differentially methylated region (DMR) of imprinted genes differed by tumor histology and location in a subset of the samples $(\mathrm{N}=41)$ (Table 4). The majority of germinomas had lower methylation than expected for an imprinted gene $(<33 \%)$ at loci that are normally methylated on both the paternal and maternal allele. Methylation patterns in teratomas were dependent on tumor location. In ovarian teratomas, loci that are typically methylated on the paternal allele had reduced methylation in almost all samples while loci that are typically methylated on the maternal allele had increased methylation. In contrast, with the exception of H19 CTCF6, the majority of extragonadal teratomas in both males and females had methylation levels in the normal range for an imprinted locus (33-66\%). This was consistent for both mature and immature teratomas (data not shown). The results for YST were more variable, with some samples exhibiting normal methylation levels at all loci while others had either reduced or increased methylation.

Table 3 Significantly enriched pathways with reduced methylation in immature teratomas compared with mature teratomas

\begin{tabular}{lll}
\hline Ingenuity Canonical Pathway & Genes & p-value \\
\hline Role of Oct4 in Mammalian Embryonic Stem Cell Pluripotency & SOX2,CASP6,SPP1,BMI1,RARA,PARP1 & 0.0015 \\
\hline Axonal Guidance Signaling & GLI2,BMP4,BDNF,BMP2,PIK3R1,EGF,VEGFB,KRAS,LIMK1,PTCH2, \\
& EPHB1,GLI,,NGFR,DCC,EFNB3,ERBB2,ITGB1,TUBB3,WNT2B,MMP10, \\
EPHA3,PDGFB,NTRK2,EPHA5,EPHA2 & 0.0045 \\
\hline Human Embryonic Stem Cell Pluripotency & BMP4,BDNF,BMP2,PIK3R1,FGFR1,WNT2B,TDGF1,FGFR2,PDGFB, \\
\hline PAK Signaling & APC,SOX2,FGFR3,NTRK2,PDGFRA,CTNNB1,PDGFRB \\
\hline PDGF Signaling & ITGB1,MYLK,PIK3R1,PDGFRA,KRAS,EPHA3,TNF,PDGFB,PDGFRB,LIMK1 & 0.01 \\
\hline NF-KB Signaling & ABL2,PIK3R1,MAP3K1,PDGFRA,CAV1,KRAS,EIF2AK2,PDGFB,PDGFRB & 0.01 \\
\hline PTEN Signaling & MAP2K6,BMP4,BMP2,PIK3R1,FGFR1,EGF,FGFR2,KRAS,DDR1,FGFR3, \\
\hline Transcriptional Regulatory Network in Embryonic Stem Cells & NTRK2,NGFR,KDR,INS,PDGFRA,EIF2AK2,TNF,PDGFRB \\
\hline Estrogen Biosynthesis & ITGB1,PIK3R1,FGFR1,FGFR2,KRAS,CCND1,DDR1,FGFR3,NTRK2, \\
\hline HER-2 Signaling in Breast Cancer & NGFR,KDR,PDGFRA,PDGFRB \\
\hline Gap Junction Signaling & SOX2,ISL1,PAX6 \\
\hline Actin Cytoskeleton Signaling & CYP2E1,HSD17B12,CYP1B1 \\
\hline Embryonic Stem Cell Differentiation into Cardiac Lineages & ITGB1,PIK3R1,EGF,KRAS,ERBB3,ERBB2,CCND1,AREG/AREGB \\
\hline
\end{tabular}


Table 4 Methylation in imprinted genes by tumor location and histology

\begin{tabular}{|c|c|c|c|c|c|c|}
\hline \multirow{4}{*}{ Average Methylation ${ }^{1}$} & \multicolumn{4}{|c|}{ Females } & \multicolumn{2}{|c|}{ Males } \\
\hline & \multirow[t]{2}{*}{ YST } & \multirow[t]{2}{*}{ Germinoma } & \multicolumn{2}{|c|}{ Teratoma } & \multirow[t]{2}{*}{ YST } & \multirow[t]{2}{*}{ Teratoma } \\
\hline & & & Ovarian & Extragonadal & & \\
\hline & $\mathrm{N}^{2}(\%)$ & $\mathrm{N}^{2}(\%)$ & $\mathrm{N}^{2}(\%)$ & $\mathrm{N}^{2}(\%)$ & $\mathrm{N}^{2}(\%)$ & $\mathrm{N}^{2}(\%)$ \\
\hline
\end{tabular}

Paternal Allele Methylated

\section{H19 CTCF3}

\begin{tabular}{|c|c|c|c|c|c|c|}
\hline$<33 \%$ & $2(25)$ & $4(67)$ & $9(90)$ & $1(12)$ & $2(50)$ & $1(25)$ \\
\hline $33-66 \%$ & $6(75)$ & $2(33)$ & $1(10)$ & 7 (88) & $1(25)$ & $3(75)$ \\
\hline \multirow[t]{2}{*}{$>66 \%$} & 0 & 0 & 0 & 0 & $1(25)$ & 0 \\
\hline & & & & 0.003 & & \\
\hline \multicolumn{7}{|c|}{ H19 CTCF6 } \\
\hline$<33 \%$ & $4(50)$ & $5(83)$ & $9(90)$ & $2(29)$ & $2(50)$ & $3(75)$ \\
\hline $33-66 \%$ & $4(50)$ & $1(17)$ & $1(10)$ & $5(71)$ & $2(50)$ & $1(25)$ \\
\hline \multirow[t]{2}{*}{$>66 \%$} & 0 & 0 & 0 & 0 & 0 & 0 \\
\hline & & & & 0.04 & & \\
\hline \multicolumn{7}{|l|}{ IGF2 } \\
\hline$<33 \%$ & $2(29)$ & $4(67)$ & $8(80)$ & $1(12)$ & 0 & 0 \\
\hline $33-66 \%$ & $5(71)$ & $2(33)$ & $2(20)$ & 7 (88) & $4(100)$ & $4(100)$ \\
\hline \multirow[t]{3}{*}{$>66 \%$} & 0 & 0 & 0 & 0 & 0 & 0 \\
\hline & & & & 0.02 & & \\
\hline & Materr & thylated & & & & \\
\hline \multicolumn{7}{|l|}{ KvDMR } \\
\hline$<33 \%$ & $2(25)$ & $4(67)$ & 0 & $3(37)$ & $3(75)$ & $1(25)$ \\
\hline $33-66 \%$ & $6(75)$ & $2(33)$ & $2(20)$ & $5(63)$ & 0 & $3(75)$ \\
\hline \multirow[t]{2}{*}{$>66 \%$} & 0 & 0 & $8(80)$ & 0 & $1(25)$ & 0 \\
\hline & & & & $<0.0001$ & & \\
\hline \multicolumn{7}{|l|}{ PEG3 } \\
\hline$<33 \%$ & 0 & 6 100) & 0 & $1(12)$ & 0 & 0 \\
\hline $33-66 \%$ & $6(75)$ & 0 & $1(10)$ & 7 (88) & 1 (33) & $4(100)$ \\
\hline \multirow[t]{2}{*}{$>66 \%$} & $2(25)$ & 0 & $9(90)$ & 0 & $2(67)$ & 0 \\
\hline & & & & $<0.0001$ & & \\
\hline \multicolumn{7}{|l|}{ SNRPN } \\
\hline$<33 \%$ & $3(38)$ & $6(100)$ & $1(10)$ & $2(25)$ & $4(100)$ & 0 \\
\hline $33-66 \%$ & $5(62)$ & 0 & 0 & $6(75)$ & 0 & $4(100)$ \\
\hline$>66 \%$ & 0 & 0 & $9(90)$ & 0 & 0 & 0 \\
\hline
\end{tabular}

${ }^{1}$ Categories represent three methylation states based on the average percent methylation across all CpG loci analyzed in the DMR: $<33 \%$ (hypomethylation), $33-66$ $\%$ (median methylation), and $>66 \%$ methylation (hypermethylation).

${ }^{2}$ N's do not sum to total due to missing data.

We also compared methylation at imprinted loci in normal and tumor tissue in the 5 samples with adjacent normal DNA (Table 5). With a few exceptions, the normal adjacent tissue exhibited DNA methylation within the expected range (34 - 66\% methylation) in samples where the tumor tissues were outside the expected range $(0-33 \%$ or $>66 \%$ methylation).

\section{Discussion}

We identified differential methylation by tumor histology in a series of pediatric GCTs, with evidence that YSTs exhibit promoter hypermethylation in a large number of cancer-related genes while germinomas and teratomas do not. These $\mathrm{CpG}$ loci were not hypermethylated in the normal adjacent tissue from two patients with YSTs, suggesting that methylation patterns 
Table 5 Average methylation at imprinted genes in five samples with paired normal adjacent tissue

\begin{tabular}{|c|c|c|c|c|c|}
\hline & $\begin{array}{c}\text { Ovarian Germinoma } \\
\text { Age } 7 \\
\text { Average Methylation }^{1}\end{array}$ & $\begin{array}{c}\text { Ovarian Teratoma } \\
\text { Age } 21 \\
\text { Average Methylation }{ }^{1}\end{array}$ & $\begin{array}{c}\text { Ovarian YST } \\
\text { Age } 19 \\
\text { Average Methylation }^{1}\end{array}$ & $\begin{array}{l}\text { Ovarian Germinoma } \\
\text { Age } 11 \\
\text { Average Methylation }^{1}\end{array}$ & $\begin{array}{c}\text { Testicular YST } \\
\text { Age } 1 \\
\text { Average Methylation }^{1}\end{array}$ \\
\hline \multicolumn{6}{|c|}{ H19 CTCF3 } \\
\hline Normal & $48 \%$ & $48 \%$ & $61 \%$ & $49 \%$ & $50 \%$ \\
\hline Tumor & $13 \%$ & $8 \%$ & $63 \%$ & $14 \%$ & $26 \%$ \\
\hline
\end{tabular}

\begin{tabular}{llllll}
\hline H19 CTCF6 & & & & & \\
\hline Normal & $36 \%$ & $36 \%$ & $44 \%$ & $34 \%$ & \\
\hline Tumor & $10 \%$ & $6 \%$ & $39 \%$ & $10 \%$ & \\
\hline & & & & & \\
\hline IGF2 & & & $68 \%$ & $31 \%$ & $63 \%$ \\
\hline Normal & $54 \%$ & $53 \%$ & $26 \%$ & $9 \%$ & $55 \%$ \\
\hline Tumor & $12 \%$ & $33 \%$ & & & \\
\hline
\end{tabular}

\begin{tabular}{lllllll}
\hline KvDMR & & & & & \\
\hline Normal & $56 \%$ & $54 \%$ & $78 \%$ & $53 \%$ & $63 \%$ & $1 \%$ \\
\hline Tumor & $11 \%$ & $97 \%$ & $24 \%$ & $10 \%$ & \\
\hline
\end{tabular}

\begin{tabular}{llllll}
\hline SNRPN & & & & \\
\hline Normal & $41 \%$ & $37 \%$ & $55 \%$ & $N^{2}$ & $21 \%$ \\
\hline Tumor & $10 \%$ & $81 \%$ & $19 \%$ & $40 \%$ & $2 \%$ \\
\hline
\end{tabular}

\section{PEG3}

\begin{tabular}{lccccc}
\hline Normal & $34 \%$ & $39 \%$ & $54 \%$ & $\mathrm{NA}^{2}$ & $37 \%$ \\
\hline Tumor & $9 \%$ & $86 \%$ & $88 \%$ & $40 \%$ & $\mathrm{NA}^{2}$ \\
\hline
\end{tabular}

${ }^{1}$ Average percent methylation across all CpG loci analyzed in the DMR.

${ }^{2} \mathrm{NA}$ : Sample failed to amplify.

also distinguish yolk sac tumor tissue from normal ovary or testis tissue. Four pathways, most notably a human embryonic stem cell pathway, were over-represented among the CpG loci that were hypermethylated in YSTs. A smaller number of $\mathrm{CpG}$ loci exhibited significantly different methylation in a comparison of mature and immature teratomas, however these loci were strikingly enriched for genes associated with embryonic stem cell pluripotency and developmental signaling pathways, such as PTEN, PDGF and NF- $\kappa$ B. In addition, immature teratomas were enriched for differential methylation of genes involved in axonal guidance signaling, reflecting the neuroepithelial character of these tumors. We also saw differences in global methylation at LINE1 elements and in methylation at imprinted loci by tumor location and histology.

Our results are consistent with the few studies to date that have evaluated promoter hypermethylation in pediatric GCT. Promoter hypermethylation has been identified in three tumor suppressor genes (APC [6], RUNX3 [7] and HIC1 [8]) in a sample of 10 infant testicular YSTs. Furukawa et al. [5] found differences in methylation levels in 2 imprinted genes and 17 tumor suppressor genes by tumor histology, with abnormal epigenetic reprogramming occurring in YSTs but not in seminomas or teratomas. In a more recent study, Jeyapalan et al. [9] evaluated both global hypomethylation of LINE-1 elements and promoter specific hypermethylation using the Illumina GoldenGate Cancer Methylation Panel in germinomas and YST (this study did not include teratomas). They found evidence for global hypomethylation in both histologic subtypes of GCT, while promoter hypermethylation was identified only in YST. Jeyapalan et al. [9] identified a list of 33 genes that were hypermethylated in more than $80 \%$ of YSTs and in $<25 \%$ of germinomas. Of these 33 genes, all exhibited significantly increased methylation in the YSTs in our series, with 12 included in the list of $23 \mathrm{CpG}$ loci with greater than 2.75 fold increased methylation in YSTs (Table 2). This hypermethylator phenotype in YSTs was previously reported to be associated with increased expression of DNMT3B [9].

Histologic characteristics of GCTs are dependent on the degree of differentiation that has occurred at the 
time of transformation [27,28]. Cells that do not undergo differentiation following transformation become germinomas. According to one model, neoplastic cells that undergo differentiation become embryonal carcinomas followed by further differentiation into embryonic (teratomas) or extra-embryonic (choriocarcinoma or YSTs) tumors. In addition, the partial erasure of methylation at imprinted genes in pediatric GCTs suggests that they originate from a germ cell at an earlier stage of development than adult TGCTs, which have complete erasure of methylation at imprinted genes [28]. Despite this difference, studies in adult GCTs have identified methylation differences by tumor histology similar to studies in the pediatric age group. Analyses of methylation in selected tumor suppressor genes [29-31] or global methylation profiles $[4,32]$ have identified increased methylation in non-seminomas, including GCTs with a YST component, compared to seminomas. At this time, it is unclear whether these methylation differences by tumor stage are driving tumor potential of the GCT, or if they reflect the stage of normal embryonic development of the germ cell when transformation occurred.

The lack of a difference in promoter methylation in teratomas and dysgerminomas was somewhat surprising, given that dysgerminomas are undifferentiated while teratomas are differentiated. We did, however, observe differences in global methylation of LINE1 elements in teratomas compared with dysgerminomas. Consistent with our findings, studies of methylation using $5{ }^{\mathrm{m}} \mathrm{C}$ staining [2] and restriction landmark genomic scanning [1] in adult testicular GCTs have reported that seminomas exhibit global hypomethylation while nonseminomas exhibit widespread methylation. The study by Jeyapalan et al. [9] did not include teratomas; however, consistent with our findings, the germinomas could not be distinguished from the normal tissue samples included on the array and did not exhibit promoter hypoor hypermethylation. They did also observe global hypomethylation in LINE-1 elements in both the germinomas and the YSTs, consistent with previous data suggesting that methylation of global repetitive elements does not always correlate with methylation in the promoter region of genes [33].

Previous studies have detected alterations in imprinting in cancer in both adult and pediatric tumors, including GCTs [10-12] Genomic imprinting is an epigenetic phenomenon (driven by methylation) that results in parent-of-origin specific gene expression. Because PGCs erase their inherited imprint and re-establish the correct sex-specific imprint following arrival on the genital ridge, loss of imprinting (LOI) has been proposed as a marker for the stage of PGC development when the tumor arose [34-37]. Several years ago, we evaluated genomic imprinting of H19/IGF2 in 11 informative tumors from this set of pediatric GCTs [38] and found that LOI occurred in pediatric GCTs. These observations were supported by the findings of an additional small study of imprinting in pediatric GCTs [10]. Here, we evaluated methylation in imprinted genes rather than allele specific expression in order to increase the number of samples with informative results, and we expanded the analysis to include three genes that are typically methylated on the maternal allele. We found significant differences in methylation levels at imprinted loci by tumor histology and location. As expected based on a previous analysis of H19/IGF2 methylation in adult and pediatric GCTs [11], the germinomas exhibited hypomethylation at all loci. Methylation at imprinted loci also distinguished teratomas located in the ovary vs. extragonadal locations, with ovarian teratomas exhibiting hypomethylation at CpG loci typically methylated on the paternal allele and hypermethylation at CpG loci typically methylated on the maternal allele. This finding provides further evidence that ovarian teratomas are parthenogenetic in origin $[15,39,40]$. Overall, these data provide direct support for the theory that methylation status at imprinted loci in GCTs represents the origin and stage of development of the PGC when transformation occurred [28].

This study population represents a well-characterized sample of pediatric GCTs including samples from all three major histologic subgroups; however, several limitations must be considered when interpreting the results. The number of germinomas was relatively small and consisted only of samples from females, which may have limited our ability to detect differences in methylation in this group. The lack of age-matched normal germ cell tissue limited our ability to detect methylation differences between GCTs and normal germ cells. We also evaluated methylation only in the $\mathrm{CpG}$ loci of a relatively small number of genes that have previously been implicated in cancer. A more unbiased evaluation of genome wide methylation would provide a more comprehensive picture of methylation patterns in GCTs.

\section{Conclusion}

These data demonstrate a distinct methylation pattern in YSTs compared to germinomas and teratomas, consisting of hypermethylation at a large number of genes known to be involved in tumorigenesis. The CpG loci identified as hypermethylated in YSTs included in our study overlapped remarkably with the CpG loci identified in two independent series of YSTs recently reported by Jeyapalan et al. [9]. Whether these alterations result from exposure to environmental agents in utero or simply are a result of abnormal PGC development remains to be elucidated. Further analyses will be required to better understand the functional and therapeutic consequences of this altered methylation signature. 


\section{Additional file}

Additional file 1: Table S1. Comparison of Methylation and gene expression. Table S2. Wnt pathway gene list. Table S3. Average Ct. Table S4. Average Delta(Ct). Table S5. $2 \wedge(-A v g .(D e l t a(C t))$. Table S6. Fold Change. Table S7. p-value. Table S8. Fold-Regulation. Table S9. Expression of SOX2 and DNMT3B by tumor histology.

\section{Abbreviations}

GCT: Germ cell tumor; GER: Germinoma; YST: Yolk sac tumor; IT: Immature teratoma; RPMM: Recursively partitioned mixture model; LINE1: Long interspersed noncoding element 1 .

\section{Competing interests}

The authors declare that they have no competing interests.

\section{Authors' contributions}

JA conceived of the study and participated in its design and coordination, examined tumor specimens, analyzed data and wrote the manuscript. JAR conceived of the study and participated in its design and coordination. BC analyzed tumor methylation data and application of the RPMM. NJF and KSC examined tumor specimens, prepared samples for analysis and analyzed data. AJH, HN and JKK performed methylation array analysis. DR and ALF provided expert consultation on tumor histology and helped to draft the manuscript. JNP conceived of the study and participated in its design and coordination, examined tumor specimens, analyzed data and wrote the manuscript. All authors read and approved the final manuscript.

\section{Acknowledgments}

The authors would like to acknowledge the Biomedical Genomics Center at the University of Minnesota for performing the DNA methylation analysis. Supported by grants from the National Institutes of Health (R01 CA135731 to J.F.A., R03 CA141481 to J.N.P., K05 CA157439 to J.A.R.); the Minnesota Medical Foundation; the Cancer Prevention and Research Institute of Texas (RP110394 to J.F.A.); the Amon G. Carter Foundation, Fort Worth, TX; and the Children's Cancer Research Fund, Minneapolis, MN

\section{Author details}

'Department of Pediatrics, University of Texas Southwestern Medical Center, Dallas, TX 75390, USA. ²Department of Molecular Biology, University of Texas Southwestern Medical Center, Dallas, TX 75390, USA. ${ }^{3}$ Department of Pathology, University of Texas Southwestern Medical Center, Dallas, TX 75390, USA. ${ }^{4}$ Center for Cancer and Blood Disorders, Children's Medical Center, Dallas, TX 75390, USA. ${ }^{5}$ Department of Pediatrics, Division of Pediatric Epidemiology and Clinical Research, Minneapolis, MN 55455, USA. ${ }^{6}$ Masonic Cancer Center, University of Minnesota, Minneapolis, MN 55455, USA. ${ }^{7}$ Division of Epidemiology and Community Health, University of Minnesota, Minneapolis, MN 55455, USA. ${ }^{8}$ Department of Community and Family Medicine, Section of Biostatistics and Epidemiology, Dartmouth Medical School, Hanover, NH 03755, USA. ${ }^{9}$ Dana Farber Cancer Institute, Boston 02115MA, USA.

Received: 25 October 2012 Accepted: 14 June 2013

Published: 27 June 2013

\section{References}

1. Smiraglia DJ, Szymanska J, Kraggerud SM, Lothe RA, Peltomaki P, Plass C: Distinct epigenetic phenotypes in seminomatous and nonseminomatous testicular germ cell tumors. Oncogene 2002, 21(24):3909-3916.

2. Netto GJ, Nakai Y, Nakayama M, Jadallah S, Toubaji A, Nonomura N, Albadine R, Hicks JL, Epstein Jl, Yegnasubramanian S, et al: Global DNA hypomethylation in intratubular germ cell neoplasia and seminoma, but not in nonseminomatous male germ cell tumors. Mod Pathol 2008, 21(11):1337-1344.

3. Bussey KJ, Lawce HJ, Himoe E, Shu XO, Heerema NA, Perlman EJ, Olson SB, Magenis RE: SNRPN methylation patterns in germ cell tumors as a reflection of primordial germ cell development. Genes Chromosomes Cancer 2001, 32(4):342-352.

4. Wermann H, Stoop H, Gillis AJ, Honecker F, Van Gurp RJ, Ammerpohl O, Richter J, Oosterhuis JW, Bokemeyer C, Looijenga LH: Global DNA methylation in fetal human germ cells and germ cell tumours: association with differentiation and cisplatin resistance. J Patho/ 2010, 221(4):433-442.

5. Furukawa S, Haruta M, Arai Y, Honda S, Ohshima J, Sugawara W, Kageyama Y, Higashi Y, Nishida K, Tsunematsu Y, et al: Yolk sac tumor but not seminoma or teratoma is associated with abnormal epigenetic reprogramming pathway and shows frequent hypermethylation of various tumor suppressor genes. Cancer Sci 2009, 100(4):698-708.

6. Kato N, Shibuya H, Fukase M, Tamura G, Motoyama T: Involvement of adenomatous polyposis coli (APC) gene in testicular yolk sac tumor of infants. Hum Pathol 2006, 37(1):48-53.

7. Kato N, Tamura G, Fukase M, Shibuya H, Motoyama T: Hypermethylation of the RUNX3 gene promoter in testicular yolk sac tumor of infants. Am J Pathol 2003, 163(2):387-391.

8. Rathi A, Virmani AK, Harada K, Timmons CF, Miyajima K, Hay RJ, Mastrangelo D, Maitra A, Tomlinson GE, Gazdar AF: Aberrant methylation of the HIC1 promoter is a frequent event in specific pediatric neoplasms. Clin Cancer Res 2003, 9(10 Pt 1):3674-3678.

9. Jeyapalan JN, Noor DA, Lee SH, Tan CL, Appleby VA, Kilday JP, Palmer RD, Schwalbe EC, Clifford SC, Walker DA, et al: Methylator phenotype of malignant germ cell tumours in children identifies strong candidates for chemotherapy resistance. Br J Cancer 2011, 105(4):575-585.

10. Schneider DT, Schuster AE, Fritsch MK, Hu J, Olson T, Lauer S, Gobel U, Perlman EJ: Multipoint imprinting analysis indicates a common precursor cell for gonadal and nongonadal pediatric germ cell tumors. Cancer Res 2001, 61(19):7268-7276.

11. Sievers S, Alemazkour K, Zahn S, Perlman EJ, Gillis AJ, Looijenga LH, Gobel U, Schneider DT: IGF2/H19 imprinting analysis of human germ cell tumors (GCTs) using the methylation-sensitive single-nucleotide primer extension method reflects the origin of GCTs in different stages of primordial germ cell development. Genes Chromosomes Cancer 2005, 44(3):256-264

12. Kawakami T, Zhang C, Okada Y, Okamoto K: Erasure of methylation imprint at the promoter and CTCF-binding site upstream of $\mathrm{H} 19$ in human testicular germ cell tumors of adolescents indicate their fetal germ cell origin. Oncogene 2006, 25(23):3225-3236.

13. Gobel U, Schneider DT, Calaminus G, Haas RJ, Schmidt P, Harms D: Germ-cell tumors in childhood and adolescence. GPOH MAKEI and the MAHO study groups. Ann Oncol 2000, 11(3):263-271.

14. Norris HJ, Zirkin HJ, Benson WL: Immature (malignant) teratoma of the ovary: a clinical and pathologic study of 58 cases. Cancer 1976, 37(5):2359-2372

15. Ulbright TM: Gonadal teratomas: a review and speculation. Adv Anat Pathol 2004, 11(1):10-23.

16. Epelman M, Chikwava KR, Chauvin N, Servaes S: Imaging of pediatric ovarian neoplasms. Pediatr Radiol 2011, 41(9):1085-1099.

17. Park SB, Cho KS, Kim JK: CT findings of mature cystic teratoma with malignant transformation: comparison with mature cystic teratoma. Clin Imaging 2011, 35(4):294-300.

18. Bibikova M, Lin Z, Zhou L, Chudin E, Garcia EW, Wu B, Doucet D, Thomas NJ, Wang Y, Vollmer E, et al: High-throughput DNA methylation profiling using universal bead arrays. Genome Res 2006, 16(3):383-393.

19. Woodfine K, Huddleston JE, Murrell A: Quantitative analysis of DNA methylation at all human imprinted regions reveals preservation of epigenetic stability in adult somatic tissue. Epigenetics Chromatin 2011, 4(1):1.

20. Bollati V, Baccarelli A, Hou L, Bonzini M, Fustinoni S, Cavallo D, Byun HM, Jiang J, Marinelli B, Pesatori AC, et al: Changes in DNA methylation patterns in subjects exposed to low-dose benzene. Cancer Res 2007, 67(3):876-880.

21. R: A language and environment for statistical computing. http://www. r-project.org/.

22. Christensen BC, Marsit CJ, Houseman EA, Godleski JJ, Longacker JL, Zheng S, Yeh RF, Wrensch MR, Wiemels JL, Karagas MR, et al: Differentiation of lung adenocarcinoma, pleural mesothelioma, and nonmalignant pulmonary tissues using DNA methylation profiles. Cancer Res 2009, 69(15):6315-6321.

23. Houseman EA, Christensen BC, Yeh RF, Marsit CJ, Karagas MR, Wrensch M, Nelson HH, Wiemels J, Zheng S, Wiencke JK, et al: Model-based clustering of DNA methylation array data: a recursive-partitioning algorithm for high-dimensional data arising as a mixture of beta distributions. BMC Bioinforma 2008, 9:365. 
24. Christensen BC, Kelsey KT, Zheng S, Houseman EA, Marsit CJ, Wrensch MR, Wiemels JL, Nelson HH, Karagas MR, Kushi LH, et al: Breast cancer DNA methylation profiles are associated with tumor size and alcohol and folate intake. PLoS Genet 2010, 6(7):e1001043.

25. Benjamini $Y$, Hochberg $Y$ : Controlling the false discovery rate: a practical and powerful approach to multiple testing. J R Statist Soc B 1995, 57:289-300.

26. Tost J, Jammes H, Dupont JM, Buffat C, Robert B, Mignot TM, Mondon F, Carbonne B, Simeoni U, Grange G, et al: Non-random, individual-specific methylation profiles are present at the sixth CTCF binding site in the human H19/IGF2 imprinting control region. Nucleic Acids Res 2007, 35(2):701.

27. Rescorla FJ, Breitfeld PP: Pediatric germ cell tumors. Clin Imaging 1999, 23(6):257-303.

28. Oosterhuis JW, Looijenga LH: Testicular germ-cell tumours in a broader perspective. Nat Rev Cancer 2005, 5(3):210-222.

29. Koul S, Houldsworth J, Mansukhani MM, Donadio A, McKiernan JM, Reuter VE, BosI GJ, Chaganti RS, Murty W: Characteristic promoter hypermethylation signatures in male germ cell tumors. Mol Cancer 2002, 1:8.

30. Lind GE, Skotheim RI, Fraga MF, Abeler VM, Esteller M, Lothe RA: Novel epigenetically deregulated genes in testicular cancer include homeobox genes and SCGB3A1 (HIN-1). J Pathol 2006, 210(4):441-449.

31. Manton KJ, Douglas ML, Netzel-Arnett S, Fitzpatrick DR, Nicol DL, Boyd AW Clements JA, Antalis TM: Hypermethylation of the $5^{\prime} \mathrm{CpG}$ island of the gene encoding the serine protease Testisin promotes its loss in testicular tumorigenesis. Br J Cancer 2005, 92(4):760-769.

32. Cheung HH, Lee TL, Davis AJ, Taft DH, Rennert OM, Chan WY: Genomewide DNA methylation profiling reveals novel epigenetically regulated genes and non-coding RNAs in human testicular cancer. Br J Cancer 2010, 102(2):419-427.

33. De Smet C, Loriot A: DNA hypomethylation in cancer: Epigenetic scars of a neoplastic journey. Epigenetics 2010, 5:3.

34. Looijenga LH, Verkerk AJ, Dekker MC, Van Gurp RJ, Gillis AJ, Oosterhuis JW: Genomic imprinting in testicular germ cell tumours. APMIS 1998, 106(1):187-195. discussion 196-187.

35. Miura K, Obama M, Yun K, Masuzaki H, Ikeda Y, Yoshimura S, Akashi T, Niikawa N, Ishimaru T, Jinno Y: Methylation imprinting of H19 and SNRPN genes in human benign ovarian teratomas. Am J Hum Genet 1999, 65(5):1359-1367.

36. Ueda T, Abe K, Miura A, Yuzuriha M, Zubair M, Noguchi M, Niwa K, Kawase Y, Kono T, Matsuda $Y$, et al: The paternal methylation imprint of the mouse $\mathrm{H} 19$ locus is acquired in the gonocyte stage during foetal testis development. Genes Cells 2000, 5(8):649-659.

37. Kerjean A, Dupont JM, Vasseur C, Le Tessier D, Cuisset L, Paldi A, Jouannet P, Jeanpierre M: Establishment of the paternal methylation imprint of the human $\mathrm{H} 19$ and MEST/PEG1 genes during spermatogenesis. Hum Mol Genet 2000, 9(14):2183-2187.

38. Ross JA, Schmidt PT, Perentesis JP, Davies SM: Genomic imprinting of H19 and insulin-like growth factor-2 in pediatric germ cell tumors. Cancer 1999, 85(6):1389-1394.

39. Riley PA, Sutton PM: Why are ovarian teratomas benign whilst teratomas of the testis are malignant? Lancet 1975, 1(7921):1360-1362

40. Eppig JJ, Wigglesworth K, Varnum DS, Nadeau JH: Genetic regulation of traits essential for spontaneous ovarian teratocarcinogenesis in strain LT/ Sv mice: aberrant meiotic cell cycle, oocyte activation, and parthenogenetic development. Cancer Res 1996, 56(21):5047-5054.

\section{Submit your next manuscript to BioMed Central and take full advantage of:}

- Convenient online submission

- Thorough peer review

- No space constraints or color figure charges

- Immediate publication on acceptance

- Inclusion in PubMed, CAS, Scopus and Google Scholar

- Research which is freely available for redistribution

Submit your manuscript at www.biomedcentral.com/submit 